Trauma Berufskrankh 2010 · 12 [Suppl 4]:377-386 DOI 10.1007/s10039-010-1637-4

Online publiziert: 16 . September 2010

(c) Springer-Verlag 2010

\section{H. Zwipp}

Klinik und Poliklinik für Unfall- und Wiederherstellungschirurgie, Universitätsklinikum Carl Gustav Carus, Technische Universität Dresden

\title{
Korrekturosteotomien am Rück- und Mittfuß
}

Sowohl intra- als auch extraartikuläre Korrekturosteotomien sollen bei relevanten posttraumatischen Fehlstellungen im Bereich des Fußskeletts wie bei anderen großen Gelenkfrakturen der direkten bzw. indirekten Gelenkrekonstruktion dienen, um durch die wiedergewonnene Gelenkkongruenz und Reorientierung aller Ach- sen die posttraumatische Arthrose zu vermeiden oder zumindest zu verzögern.

Im Folgenden werden 5 anatomische Ebenen des Fußes bezüglich ihrer Möglichkeiten der Korrekturosteotomie besprochen, der Rückfuß mit Talus und Kalkaneus, der so genannte Mittfuß mit Chopart- und Lisfranc-Gelenk sowie der
Vorfuß mit Korrekturosteotomie der Metatarsalia.

\section{Talus}

Bei der Osteotomie des Talus handelt es sich nicht um eine geradflächige Durchtrennung des Knochens, sondern ein sub-
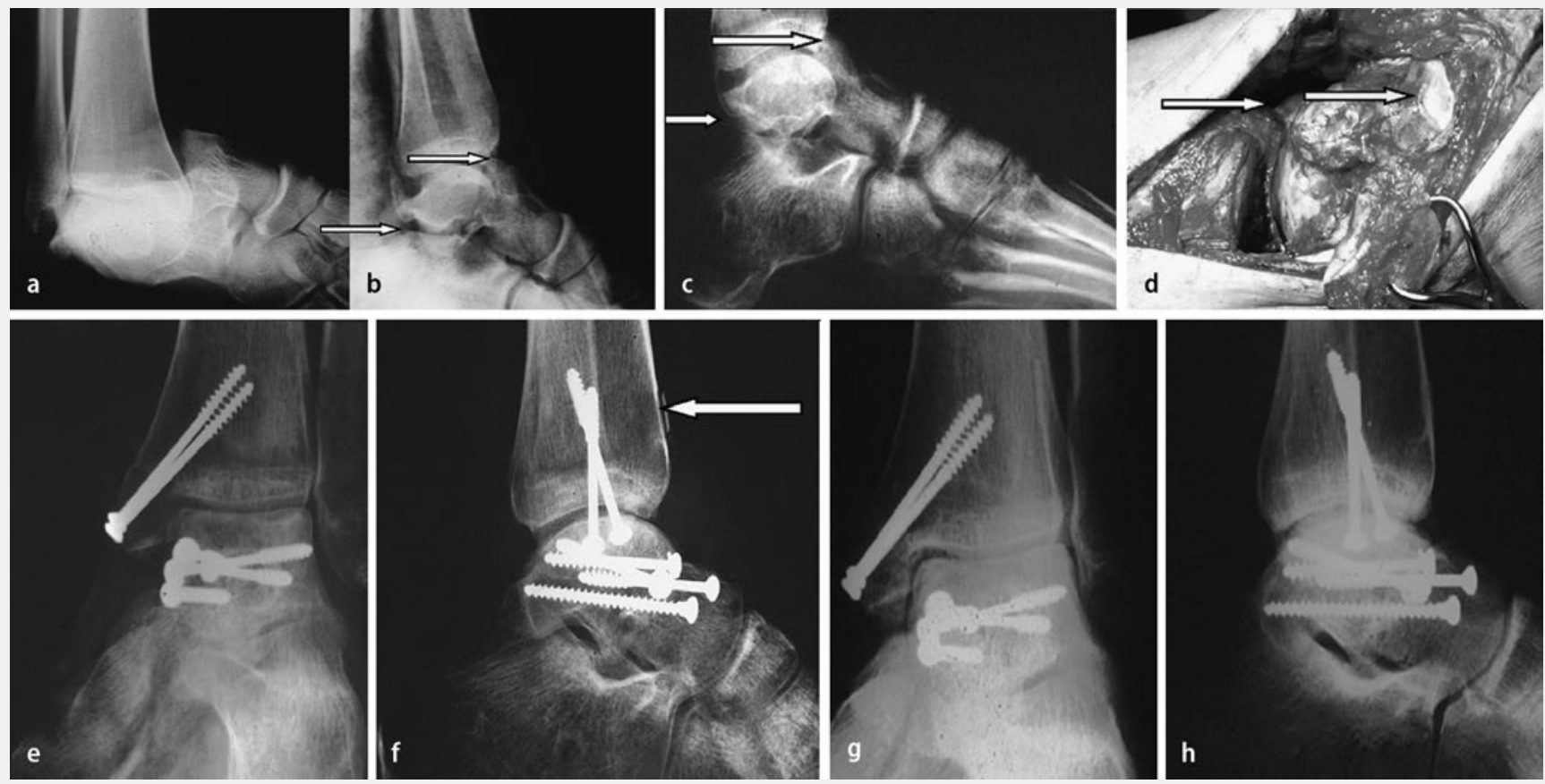

Abb. $1 \Delta$ Fallbeispiel 1: anatomische Spätrekonstruktion bei einem 21-jährigen Patienten 6 Monate nach weit dislozierter Kalkaneuskorpusfraktur im Sinne der Luxation des gesamten Fußes unter dem Talus nach medial. Die Luxationsfraktur wurde zunächst geschlossen reponiert (b) und wegen unbefriedigender Stellung tibiokalkanear für annähernd 3 Monate transfixiert, worunter es zur schwersten Fehlverheilung mit Fehlstellung im oberen Sprunggelenk und Subtalargelenk (Pfeile) kam. Eine Dorsalflexion war 6 Monate nach der Fraktur nicht möglich. Wegen der zusätzlichen Verwerfung im Subtalargelenk konnte der Patient aufgrund der Schmerzen nur mit Stützen gehen (c). Nach Osteotomie des Innenknöchels (d) und Darstellung der medialen Talusfläche zeigte sich neben der Fehlstellung im oberen Sprunggelenk auch die vermehrte Innenrotation des Taluskopfes bei varischer Fehlverheilung (Pfeil). Nach Auflösung der Frakturflächen wechselweise über den anteromedialen und anterolateralen Zugang wurde sukzessive eine anatomische Rekonstruktion möglich (e,f). Die freigelegten und reponierten Frakturflächen wurden mit 4 Schrauben unter Druck gesetzt, eine lokale Spongiosaentnahme aus der distalen Tibia (Pfeil) zur biologischen Anregung der Frakturheilung durchgeführt und der Innenknöchel mit 2 Spongiosazugschrauben $(4,0)$ fixiert. Der 3-Jahres-Follow-up ( $\mathbf{g}, \mathbf{h})$ zeigte keine nennenswerte posttraumatische Arthrose oder Partialnekrose des Talus mit einer guten Funktion des oberen Sprunggelenkes für Streckung/Beugung mit 5/0/45 


\section{Sprunggelenk und Fuß}
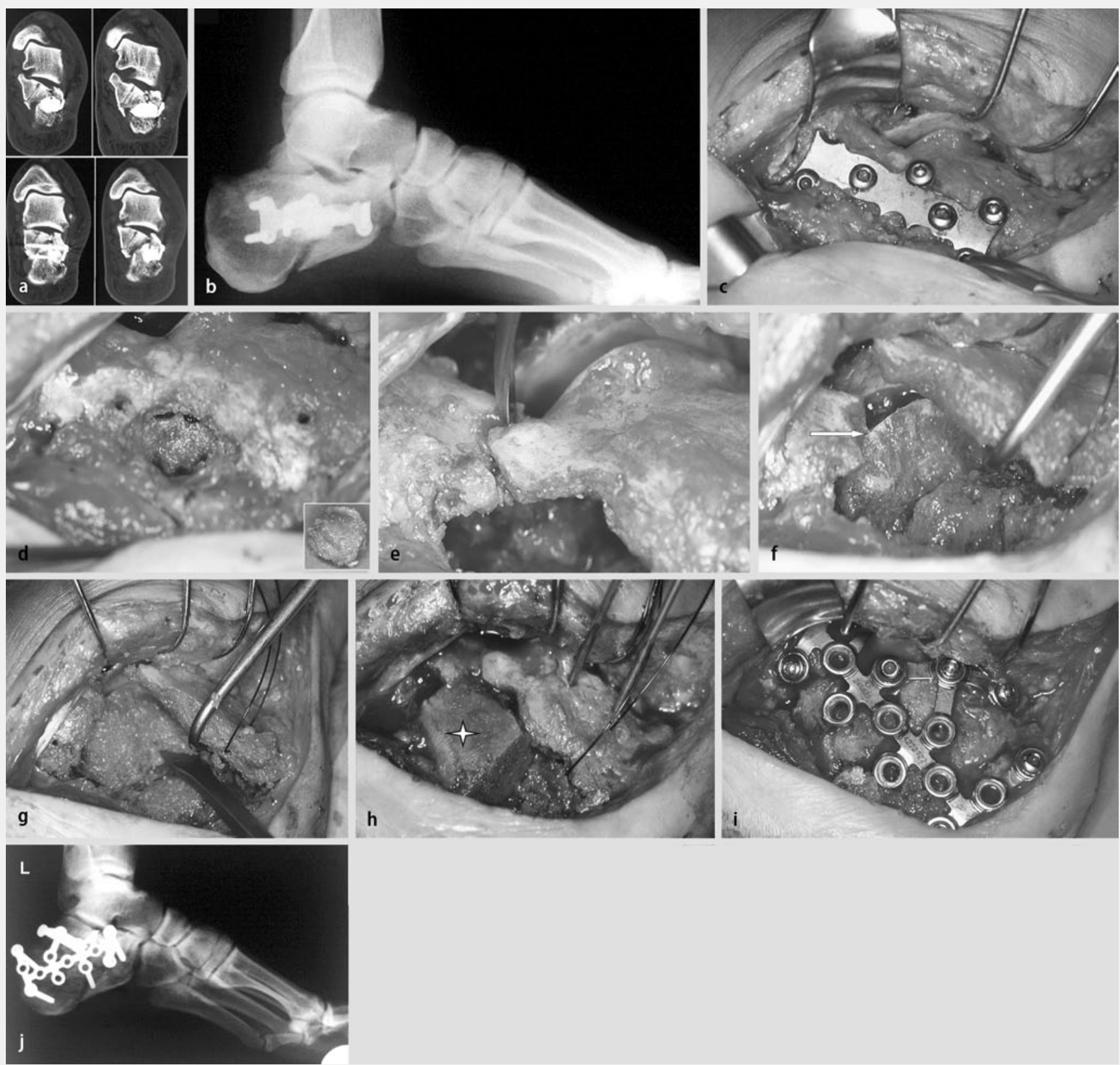

Abb. $2 \Delta$ Fallbeispiel 2: selten mögliche Subtalargelenk erhaltende Frühosteotomie der posterioren Facette des Kalkaneus bei einem 50-jährigen, chirurgisch tätigen Kollegen 7 Wochen nach Erstversorgung. In den CT-Aufnahmen (a) war die fehlende Reposition des sustentacularen Hauptfragmentes sowie des intermediären Fragmentes der posterioren Facette deutlicher erkennbar als in der seitlichen Fußaufnahme (b), welche lediglich eine Doppelkontur der posterioren Facette erkennen ließ. Nur der laterale Anteil der posterioren Facette hatte Kontakt zur Talusunterfläche, sodass trotz Endobone-Unterfütterung und Plattenosteosynthese eine unzureichende Kongruenz des Subtalargelenkes gegeben war. Aus diesem Grunde wurde die sekundäre Rekonstruktion (c-j) angestrebt. Nach Eingehen im Bereich der alten Narbe zeigte sich zunächst bei liegender Platte das Subtalargelenk im ganz lateralen Aspekt kongruent (c). Nach Entfernung des Endobone-Transplantates (d) wurden im Gissan-Winkel die alte Frakturfläche mit dem Raspatorium gelöst (e) und anschließend mit Einzinkerhaken das laterale Tuberfragment angehoben. Erst jetzt war das abgekippte Intermediärfragment (Pfeil) deutlich erkennbar (f). Durch Osteotomie mit dem Meißel (g) wurden das mediale und intermediäre Fragment gelöst. Nach Reposition und Unterfütterung mit einem kortikospongiösen Span (Stern) wurden die Fragmente temporär mit Kirschner-Drähten gehalten (h). Nach Anlegen einer winkelstabilen Platte (i) ließ die intraoperative Röntgenuntersuchung jede Doppelkontur im Bereich der posterioren Facette ausschließen (j) 
tiles Wiederauflösen fehlverheilter Frakturflächen. So ist bei Fehlverheilungen nach zentraler Talusluxationsfraktur im Sinne der "malunion“ oder „nonunion“ das oberste Ziel der Osteotomie die anatomische Wiederherstellung aller Gelenkflächen durch Auflösung der fehlverheilten Frakturflächen, um zumindest eine, wenn möglich 2 oder sogar alle 3 Gelenkebenen des Talus wiederherzustellen. Dies gelingt nach präoperativer Planung mit Röntgenbelastungsaufnahmen, CT (Computertomographie) in 2 Ebenen, evtl. mit $3 \mathrm{D}$ $\mathrm{CT}$ oder einem intraoperativen $3 \mathrm{D}-\mathrm{Mo}$ dell in der Regel nur über einen beidseitigen Zugang, d. h. medialseitig, oft mit zusätzlicher Innenknöchelosteotomie kombiniert, und lateralseitig mit einem anterolateralen oder Ollier-Zugang zum Talus.

Fallbeispiel 1 (• Abb. 1) zeigt eine solche anatomische Rekonstruktion mit Wiederherstellung aller 3 Gelenkebenen, ohne dass es durch diesen komplexen Eingriff zur Talusnekrose gekommen wäre. In 15 von 18 Fällen einer sekundär anatomisch wiederhergestellten, zentralen fehlverheilten Talusfraktur konnte die wiedergewonnene Gelenkkongruenz ohne posttraumatische Arthrose im mittleren 5-Jahres-Langzeitergebnis sichergestellt werden [4]. In einem Fall musste primär das Subtalargelenk fusioniert werden. Sekundäre Arthrodesen wurden nur einmal am oberen Sprunggelenk (OSG) nach 7 Jahren, einmal am Talonavikulargelenk nach 5 Jahren und nur in einem einzigen Fall als zweizeitige Subtalar- und OSG-Arthrodese innerhalb eines Jahres nach Rekonstruktion durchgeführt.

\section{Kalkaneus}

\section{Extraartikuläre Fehlverheilung}

Bei fehlverheilter extraartikulärer Fraktur des Kalkaneus können durch die achsenkorrigierende Osteotomie Höhe, Länge und Breite des Kalkaneus zur Wiederherstellung der Trigonometrie des Fußes in der Regel erzielt werden, sodass durch die Wiedergewinnung der biomechanischen Längenverhältnisse des $M$. triceps surae auch wieder ein kräftiger Hebelarm am Rückfuß für die physiologische Dynamik beim Gehen erreicht werden kann.

Trauma Berufskrankh 2010 - 12 [Suppl 4]:377-386 DOI 10.1007/s10039-010-1637-4

C) Springer-Verlag 2010

\section{H. Zwipp \\ Korrekturosteotomien am Rück- und Mittfuß}

\section{Zusammenfassung}

Korrekturosteotomien im Sinne der früheren Frakturflächenauflösung nach Fehlverheilung tarsaler oder metatarsaler Knochen können im günstigsten Fall die Gelenkkongruenz, so z. B. am disloziert verheilten Talus das obere Sprunggelenk, das hintere untere Sprunggelenk und gelegentlich auch zusätzlich das Talonavikulargelenk wiederherstellen, was am Kalkaneus als anatomische Spätrekonstruktion der posterioren Gelenkfacette nur selten möglich ist. In anderen Fällen können "closing wedge",,"opening wedge" oder eine translatorische Osteotomie am Kalkaneus, am Os naviculare pedis oder an den Ossa cuneiformia Varus-/Valgus- bzw. Abduktions-/Adduktionsfehlstellungen bis hin zu rotatorischen Korrekturen erzielen. Auch ei-

ne Verkürzungsosteotomie am Kuboid kann die Balance zwischen lateraler und medialer Fußsäulenlänge wiederherstellen und damit die Funktionstüchtigkeit des Fußes fördern. Klein- und Großfragmentschrauben sowie kleine, bis zu 2,0 mm messende winkelstabile Plättchen - wie zur Korrektur fehlverheilter Metatarsalia - dienen zur sicheren Ausheilung der erzielten Korrektur. Durch diese Maßnahmen können eine posttraumatische Arthrose verhindert oder deren Entwicklung zumindest verzögert werden.

\section{Schlüsselwörter}

Fehlverheilung · Pseudarthrose - Tarsus .

Metatarsus - Osteotomie

\section{Corrective osteotomy of the hind- and midfoot}

\section{Abstract}

Subtle osteotomy in the sense of disconnecting old fracture surfaces of a malunited or nonunited talus is sometimes suitable to restore secondary anatomic joint congruency of the ankle and subtalar joint, sometimes also of the talonavicular joint. Anatomic reconstruction of the posterior facet of the calcaneus can seldom be achieved. Closing or opening wedge osteotomies and shifting or rotatory osteotomies of the calcaneus and the navicular or cuneiform bones are suitable at least to correct varus or valgus deformities and abduction or adduction malpositions. Sometimes a shortening osteotomy of the cuboid can balance the lengths of the lateral and medial column. Large and small screws as well as an interlocking plate with a minimal size of $2.0 \mathrm{~mm}$ guarantee safe healing of the corrected tarsal or metatarsal bone. By anatomic secondary joint reconstruction, corrections of all axes and lengths and ensuring bone stability, post-traumatic arthritis can be prevented or its development delayed.

\section{Keywords}

Malunion - Nonunion · Tarsal bones . Metatarsal bones - Osteotomy 

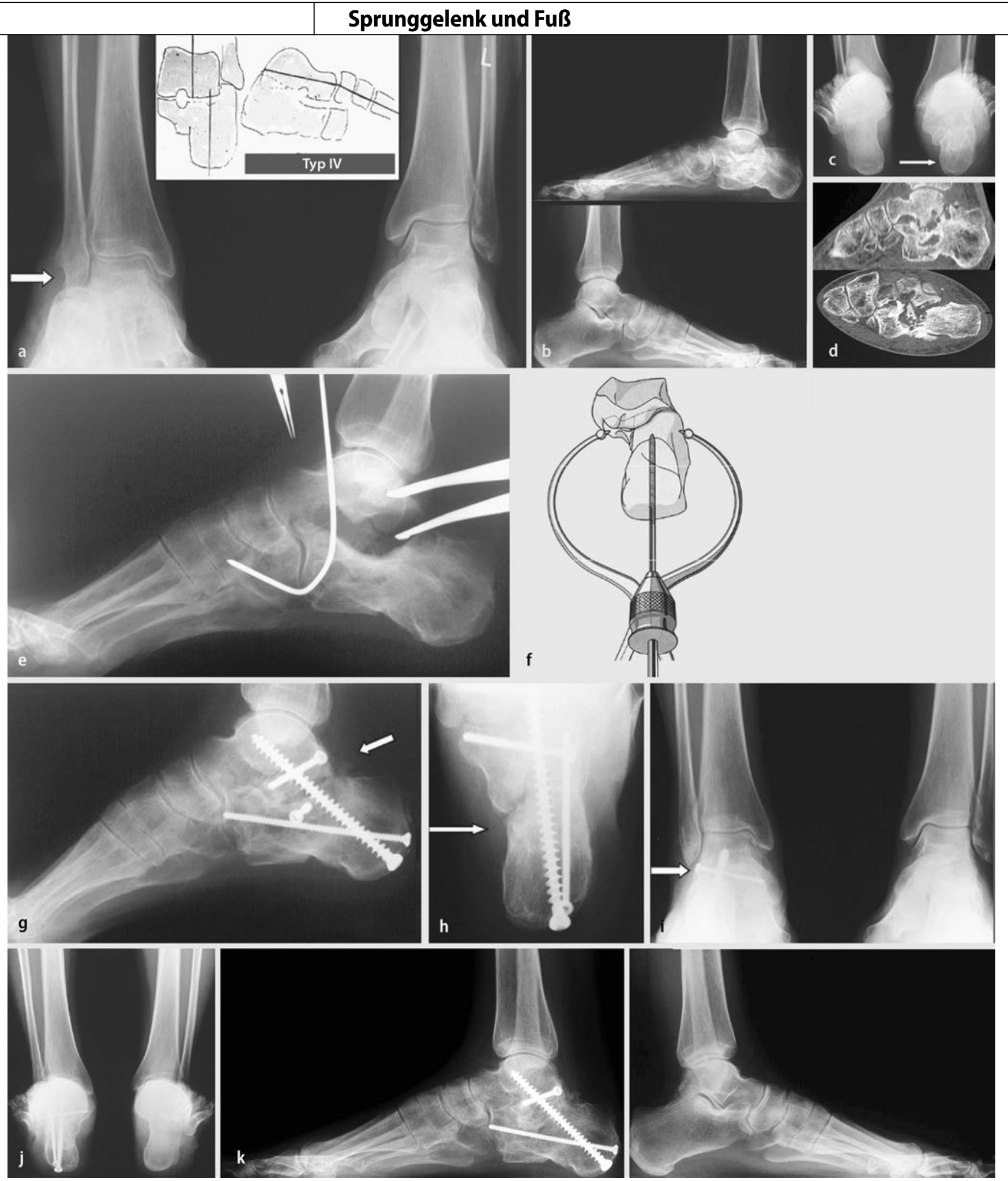

\section{Intraartikuläre Fehlverheilung}

Subtalargelenk erhaltende Osteotomien sind am Kalkaneus nur ausnahmsweise möglich, was im Fallbeispiel 2 (• Abb. 2) gezeigt wird.

\section{Intraartikuläre und luxierte Fehlverheilung (Typ IV)}

Beim Zustand nach Kalkaneusluxationsfraktur, welche konservativ oder operativ unzureichend behandelt wurde, ist in der Regel bei Fehlverheilung Typ IV und $\mathrm{V}[1,2,3]$ eine Osteotomie des Kalkaneus im Bereich der vorausgegangenen Scherfraktur über einen zusätzlichen medialen Zugang notwendig, um den weit nach lateral disloziert fehlverheilten Tuberanteil wieder zum sustentacularen Hauptfragment ausrichten zu können, d. h. damit die Rückfußachse, Höhe, Länge und Brei- 


\begin{abstract}
Abb. 34 Fallbeispiel 3: fehlverheilte, nicht ohne Osteotomie des Kalkaneus adäquat zu korrigierende Kalkaneusluxationsfraktur Typ IV. a-d präoperative Aufnahmen der 44-jährigen Patientin mit Zustand nach Luxationsfraktur des Kalkaneus links im Rahmen einer Polytraumatisierung und perkutanen Osteosynthese. a Das Tuber calcanei mit posteriorer Facette war weit nach lateral luxiert fehlverheilt und stand vollkommen unter dem selbst im Rahmen der Luxation unter Ausriss des proximalen peronäalen Sehnenretinaculums frakturierten Außenknöchel (Pfeil). b Aus den seitlichen Aufnahmen im Stehen war die erhebliche Retrokippung des Talus rechts mit Höhenverlust des Rückfußes im Seitenvergleich um etwa 1,5 cm ersichtlich. c Die operativen Saltzman-Aufnahmen zeigten den Versatz des Tubers um etwa 1,5 cm nach lateral (Pfeil). d In den CT-Aufnahmen vor Korrektur, ebenfalls etwa 1 Jahr nach Erstversorgung, imponierte die mottenfraßähnliche Zerstörung des Subtalargelenkes mit nur partieller Verheilung der lateralen Kalkaneuswand. Teilabbildungen $\mathbf{e}-\mathbf{h}$ zeigen, dass nach Osteotomie die Reposition des Tuberfragmentes nach medial nur im Sinne einer konzertierten Aktion möglich ist: Der Operateur zieht an der eingebrachten Schanz-Schraube mit Handgriff das Tuberfragment nach kaudal und hebelt mit einem von lateral eingebrachten Raspatorium mit seiner zweiten Hand gleichzeitig wie mit einem Schuhlöffel das Tuberfragment nach unten und nach medial. Der Assistent setzt nach Reposition die große Spitz-Spitz-Zange (f), um die reponierte Position zu halten. Ein initial eingebrachter Arthrodesenspreizer (e) zwischen Taluskörper und Kalkaneus zeigt in der seitlichen Röntgen-/Bildverstärkeraufnahme die notwendige Aufrichtungsachse des Taluskörpers und die Größe der jetzt einzusetzenden autologen Knochenspäne (g). Die einzubringenden Schrauben müssen quer und längs (h) die osteotomierten Frakturflächen gegeneinander stabilisieren sowie den aufgerichteten Taluskörper zum Kalkaneus in Distanz halten, weswegen 6,5er Spongiosaschrauben mit durchgehendem Gewinde gewählt wurden (g,h). Der Lateralversatz des Tubers muss zur Achskorrektur des Fußes komplett ausgeglichen sein (h). Die Halbjahreskontrollröntgenuntersuchung ergab in der OSG-a.-p.-Aufnahme im Stehen (i) den jetzt völlig freien subfibularen Raum (Pfeil). Die Saltzman-Aufnahmen des Rückfußes beidseits (j) zeigten die korrekte Rückfußachse, die seitlichen Aufnahmen im Stehen (k) die wiedergewonnene Rückfußhöhe, die ausgeglichene Retrokippung des Talus und die feste Verheilung von Osteotomie und Subtalararthrodese
\end{abstract}

te des Kalkaneus annähernd wiederherzustellen.

Fallbeispiel 3 ( $\bullet$ Abb. 3) zeigt die Fehlverheilung des rechten Kalkaneus nach Luxationsfraktur, die sich eine 44jährige PKW-Fahrerin im Rahmen eines Wegeunfalles neben zahlreichen anderen Verletzungen zugezogen hatte. Aufgrund der allgemein schlechten Situation der schwerverletzten Patientin war wegen lebenserhaltender Maßnahmen nur verzögert eine perkutane Spickdrahtosteosynthese durchgeführt worden. Durch den primären Luxationsmechanismus und die perkutan unzureichend erzielte Reposition war es zur Fehlverheilung des Tuber calcanei mit Versatz um etwa 1,5 $\mathrm{cm}$ nach lateral gekommen. Zusätzlich bestanden eine starke Retrokippung des Talus, eine Arthrose des Subtalargelenkes, eine Zerstörung des Außenknöchels mit Ausriss des peronäalen Retinaculums, eine Peronäalsehnenluxation und ein Abutment des Kalkaneus zur Fibula.

Diese schwere Fehlverheilung mit schmerzhafter Arthrose v. a. des Subtalargelenkes kann über beidseitiges Zugehen mit Osteotomie des Kalkaneus von medial über einen modifizierten McReynolds-Zugang sowie mittels eines lateralen Palmer- oder geraden epimalleolären Zuganges korrigiert werden. Nach Zurückhebeln des osteotomierten Tubers im Sinne eines konzertierten Repositionsmanövers (• Abb. 3h) zum sustentacularen Hauptfragment wurden unter temporärem Halten mit Kirschner-Drähten die Restsklerose im ehemaligen Subtalargelenkbereich mit Anheben des Taluskörpers mittels Arthrodesenspreizer (• Abb. 3e) ausgeräumt, das Tuber zum Sustentaculum verschraubt und der große Defekt mit kortikospongiösen Spänen aufgefüllt ( $\mathbf{0}$ Abb. 3f,g). AbschlieBend wurde der Tuber calcanei zum sustentacularen Fragment, zum anterioren Kalkaneus und zum Taluskörper hin im Sinne der reorientierenden subtalaren Arthrodese verschraubt ( Abb. 3f,g), wodurch Rückfußhöhe, -achse und eine stabile Ausheilung der Arthrodese gewährleistet wurden (- Abb. 3i-k).

\section{Intraartikuläre und luxierte Fehlverheilung mit Beteiligung des OSG (Typ V)}

Beim Typ V der Kalkaneusluxationsfraktur mit Fehlverheilung kommt es zusätzlich zum varischen Abkippen des Talus aus der Sprunggelenkgabel (Fallbeispiel 4). Ziel der Wiederherstellung mittels Osteotomie des Kalkaneus von medial besteht neben der Reorientierung des Rückfußes im Sinne der subtalaren Arthrodese v. a. darin, den Talus wieder in die Sprunggelenkgabel zurückzuführen, was nur durch einen dritten Zugang im Sinne des anterioren Zuganges zum oberen Sprunggelenk zwischen M.-tibialis-anterior- und M.extensor-hallucis-longus-Sehne möglich wird (• Abb. 4).

\section{Navikulare/Kuboid}

\section{Osteotomie des Os naviculare pedis}

Sie ist selten gegeben, kann aber im Rahmen eines schweren posttraumatischen Pes cavo-valgus (Fallbeispiel 5) eine sehr hilfreiche Maßnahme zur Vermeidung einer rezidivierenden pathologischen Innenrotation des Talus darstellen (s. Legende zu $\bullet$ Abb.5).

\section{Osteotomie des Kuboids}

Sie kann im Sinne der Reorientierung der 2-Säulen-Statik gelegentlich bei Komplexverletzungen des Mittfußes zur Anwendung kommen. Fallbeispiel 6 (• Abb. 6) zeigt eine drittgradig offene Chopart-/Lisfranc-Luxationsfraktur mit Zerreißung der A. dorsalis pedis und primärem Verlust größerer Anteile des Os naviculare pedis und der Ossa cuneiformia I und II. Zur Vermeidung einer plastischen Maßnahme und zur Wiederherstellung einer längenbilanzierten lateralen und medialen Fußsäule kann - wie hier im Beispiel gezeigt - eine verkürzende Osteotomie des Kuboids die Weichteilsituation lösen helfen. Der dabei vom Kuboid gewonnene Knochen kann zur Defektfüllung der fehlenden Ossa cuneiformia innerhalb der medialen Säule dienen und zusätzlich zum Längenausgleich zwischen lateraler und medialer Säule. 


\section{Sprunggelenk und Fuß}
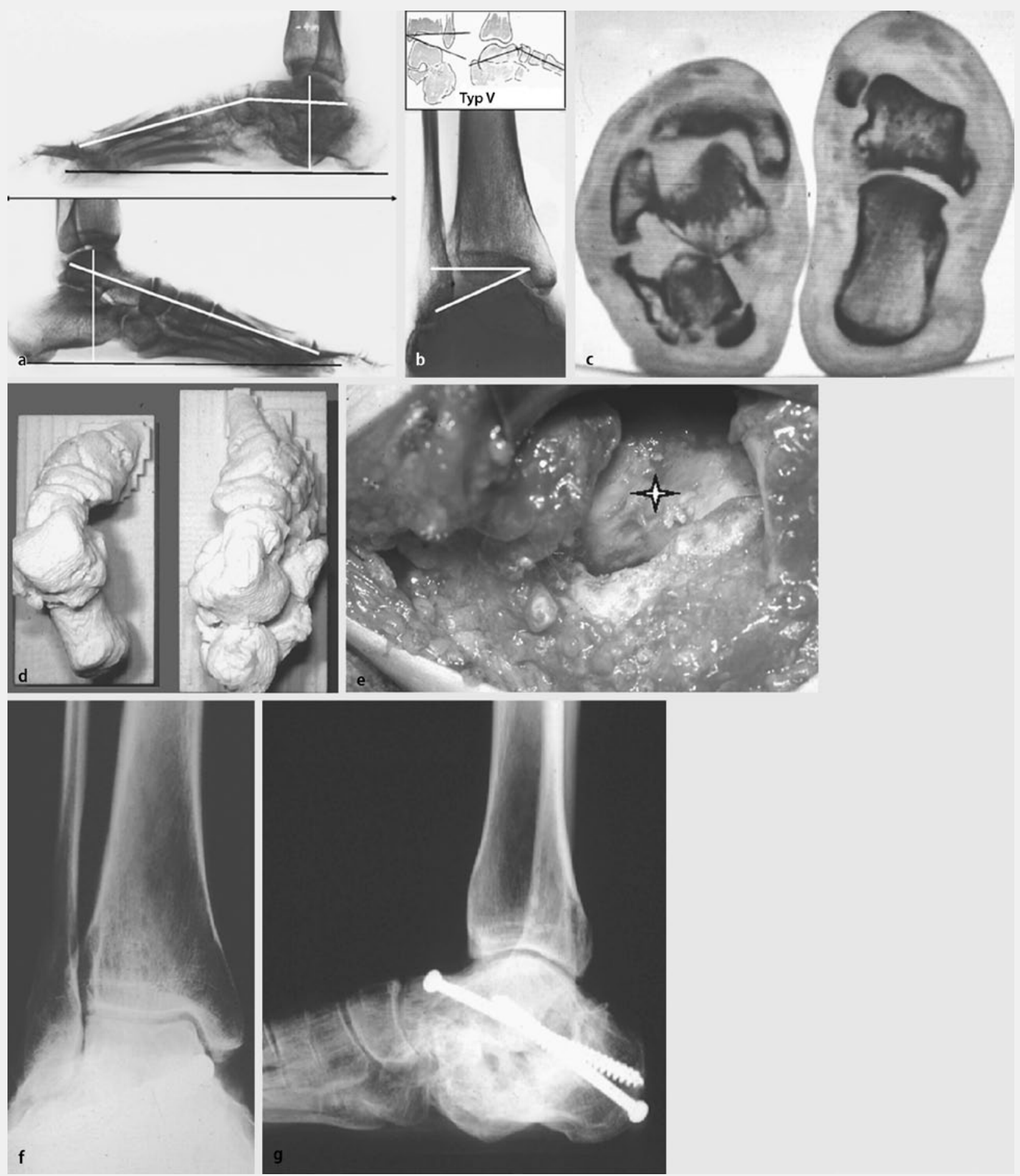

Abb. $4 \Delta$ Fallbeispiel 4: extreme Fehlverheilung einer Kalkaneusluxationsfraktur mit fehlverheilter Luxation des Taluskörpers aus der Sprunggelenkgabel 4 Monate nach Trauma bei einem 21-jährigen Patienten. Aufgrund der Schmerzhaftigkeit war der Mann an 2 Unterarmgehstützen gefesselt. Aus den Belastungsaufnahmen im Stehen $(\mathbf{a}, \mathbf{b})$ waren die erhebliche Retrokippung des Talus $\left(24^{\circ}\right)$ mit Rückfußhöhenverlust (senkrechte weiße Linie) sowie die varische Kippung des Taluskörpers um $24^{\circ}$ aus der Sprunggelenkgabel ersichtlich. Das präoperative CT (c) und das präoperative 3D-Modell (d) machten die komplexe Fehlstellung mit beträchtlicher Kippung des Talus nach lateral und Eintauchen in den erheblich verbreitert fehlverheilten, luxierten Kalkaneus, d. h. Luxation der posterioren und der kuboidalen Gelenkfläche nach lateral, deutlich. Der intraoperative Situs (e) zeigte die tief in den Kalkaneus eingesunkene Facies malleolaris lateralis tali (Stern), wobei der Knorpel selbst noch nach Freilegung gut erhalten war. Über 3 Zugänge medial und lateral zum Fersenbein sowie anterior zum oberen Sprunggelenk konnte das Sprungbein schrittweise in die Gabel zurückgeführt und der Rückfuß nach Osteotomie reorientiert und mit autologer Spongiosa und 6,5er Spongiosaschrauben im Sinne der reorientierenden Subtalararthrodese stabilisiert werden. Das 5-Jahres-Follow-up-Ergebnis (f) zeigte in beiden Ebenen das gut erhaltene obere Sprunggelenk mit verheiltem Rückfuß bei noch einliegenden Schrauben 

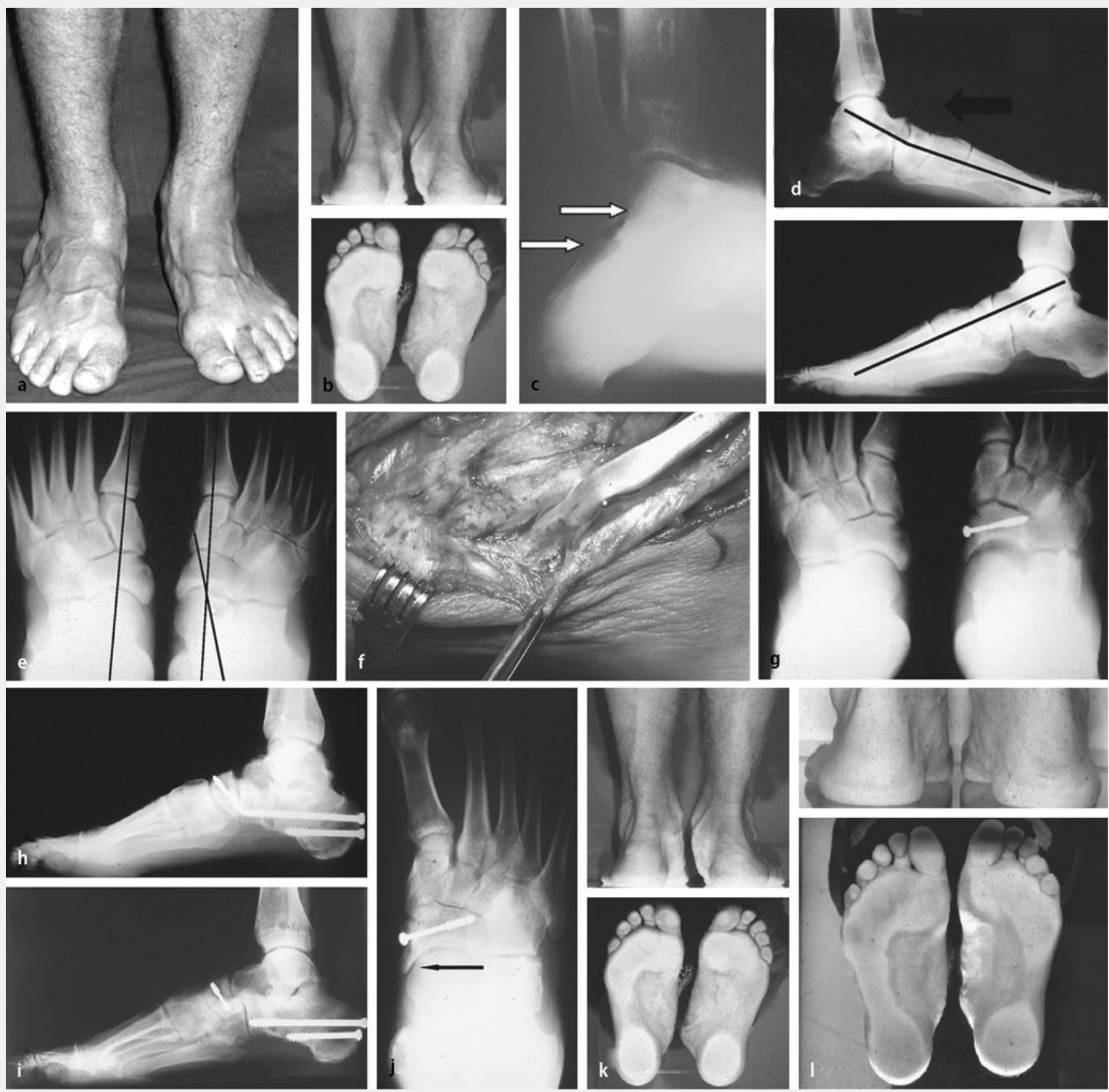

Abb. $5 \Delta$ Fallbeispiel 5: selten notwendige Osteotomie eines Os naviculare pedis bei einem 15-jährigen Patienten 1 Jahr nach ordnungsgemäßer Versorgung einer Pronations-/Abduktionsfraktur des oberen Sprunggelenkes. Die präoperativen klinischen Aufnahmen einschließlich Fotografien $(\mathbf{a}, \mathbf{b})$ zeigten den schweren posttraumatischen Pes cavo valgus rechts. In der $30^{\circ}$-Brodén-Projektion (c) war der Versatz des Kalkaneus nach lateral um $6 \mathrm{~mm}$ (weiße Pfeile) ersichtlich. Die seitlichen Belastungsaufnahmen (d) zeigten im Seitenvergleich die Inklination der talometatarsalen Achse rechts. In den dorsoplantaren Belastungsaufnahmen (e) war der Shift der Talusachse nach medial von $15^{\circ}$ zu sehen. Erst intraoperativ (f) konnte die Ursache für die schwere valgische Fehlentwicklung durch erhebliche Elongation der M.-tibialis-posterior-Sehne (hochgehalten mit einem Meniskustasthaken) und einer erkennbaren Verlängerung von mindestens $1 \mathrm{~cm}$ identifiziert werden. Die postoperativen 1-Monats-Belastungsaufnahmen zeigten in dieser frühpostoperativen Phase nach Evans-Osteotomie, varisierender Dwyer-Osteotomie und aufrichtender Osteotomie des Os naviculare pedis die anfängliche Überkorrektur ( $\mathbf{g}, \mathbf{h})$ und die spätere korrekte Ausrichtung der Cyma-Linie und der talometatarsalen Achse in beiden Ebenen nach $1 \mathrm{Jahr}(\mathbf{i} \mathbf{i} \mathbf{j})$. Durch die Osteotomie des Os naviculare wurde ein weiterer Shift des Taluskopfes nach medial unmöglich gemacht (j, schwarzer Pfeil). Die Spiegeltischaufnahme präoperativ (k) und 1 Jahr postoperativ (I) ergaben im Vergleich die fast vollständige Korrektur des schweren Rückfußvalgus 


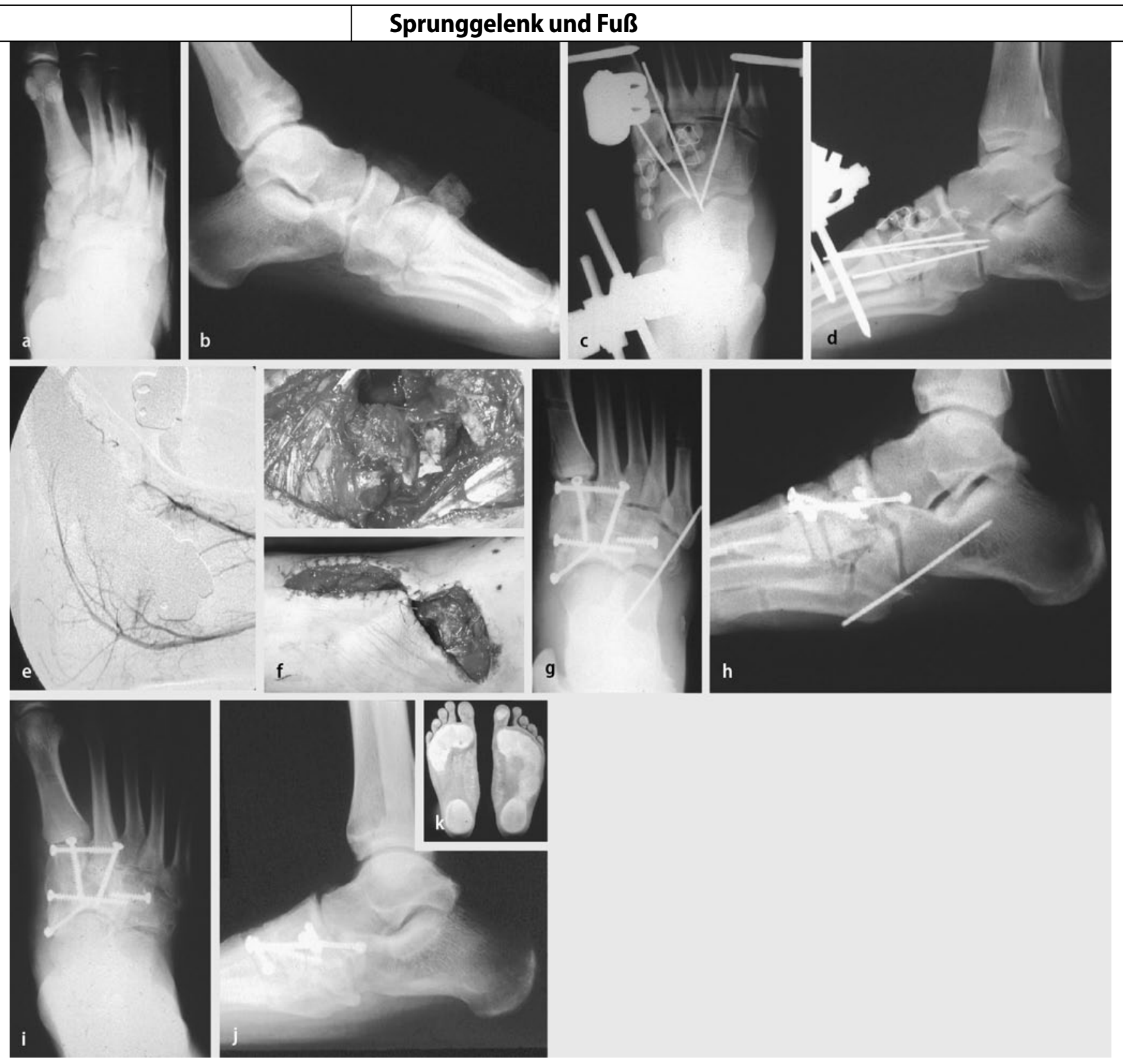

Abb. $6 \Delta$ Fallbeispiel 6: Möglichkeit einer reorientierenden verkürzenden Kuboidosteotomie bei 21-jährigem Motorradfahrer mit komplexer offener Chopart-/Lisfranc-Luxationsfraktur. Aus den präoperativen Aufnahmen $(\mathbf{a}, \mathbf{b})$ waren bei drittgradig offener Chopart-/Lisfranc-Luxationsfraktur die Dislokation des geborstenen Os naviculare pedis sowie die Defektsituation der Ossa cuneiformia I und II ersichtlich. Im ersten Schritt der intraoperativen Versorgung (c,d) wurden die Defekte mit PMMA-Kugelketten (PMMA: Polymethylmethacrylat) aufgefüllt und die Chopart- und Lisfranc-Gelenkreihe mit temporärer Transfixation mittels Kirschner-Drähten und tibiotarsaler Fixateur-externe-Transfixation reponiert. In der postoperativen Angiographie (e) wurde der komplette Abriss der A. dorsalis pedis detektiert. Aufgrund der intraoperativ im Rahmen der Second-Look-Operation (f-h) ersichtlichen relevanten Weichteildefektsituation wurde eine Verkürzungsosteotomie des Kuboids gewählt, mit Auffüllen der Defekte durch die dadurch gewonnene Spongiosa, Äquilibrierung der medialen und lateralen Fußsäulenlänge mit Schraubenosteosynthese und temporärer Transfixation der lateralen Fußsäule mittels Kirschner-Draht von der Basis des 5. Mittelfußknochens bis in den Kalkaneuskörper. Im 6-Monats-Ausheilungsergebnis (i-k) waren die Wiederherstellung des kongruenten Chopart-Gelenkes mit normal geschwungener Cyma-Linie in beiden Ebenen, Fusion der Ossa cuneiformia zum Os naviculare pedis untereinander und zur Basis des zweiten Mittelfußknochens unter mäßiger Verkürzung des Fußes (k) bei harmonischer medialer und lateraler Fußsäulenlänge nachweisbar

\section{Ossa cuneiformia}

Eine Korrekturosteotomie der Ossa cuneiformia im Sinne einer „closing-wedge“Osteotomie kann der Reorientierung der medialen Fußsäule bei Abduktionsfehl- stellung dienen, insbesondere bei zusätzlicher Verlängerung der lateralen Fußsäule mittels Osteotomie des distalen Kalkaneus und kortikospongiöser Spaninterposition im Sinne dessen Verlängerung zur
Harmonisierung beider Fußsäulenlängen (Fallbeispiel 7).

Aus - Abb. 7 sind der schwere präoperative Weichteilschaden (D Abb.7ac) und ossäre Befund ( $\bullet$ Abb. 7d) eines 8-jährigen türkischen Jungen nach Ver- 

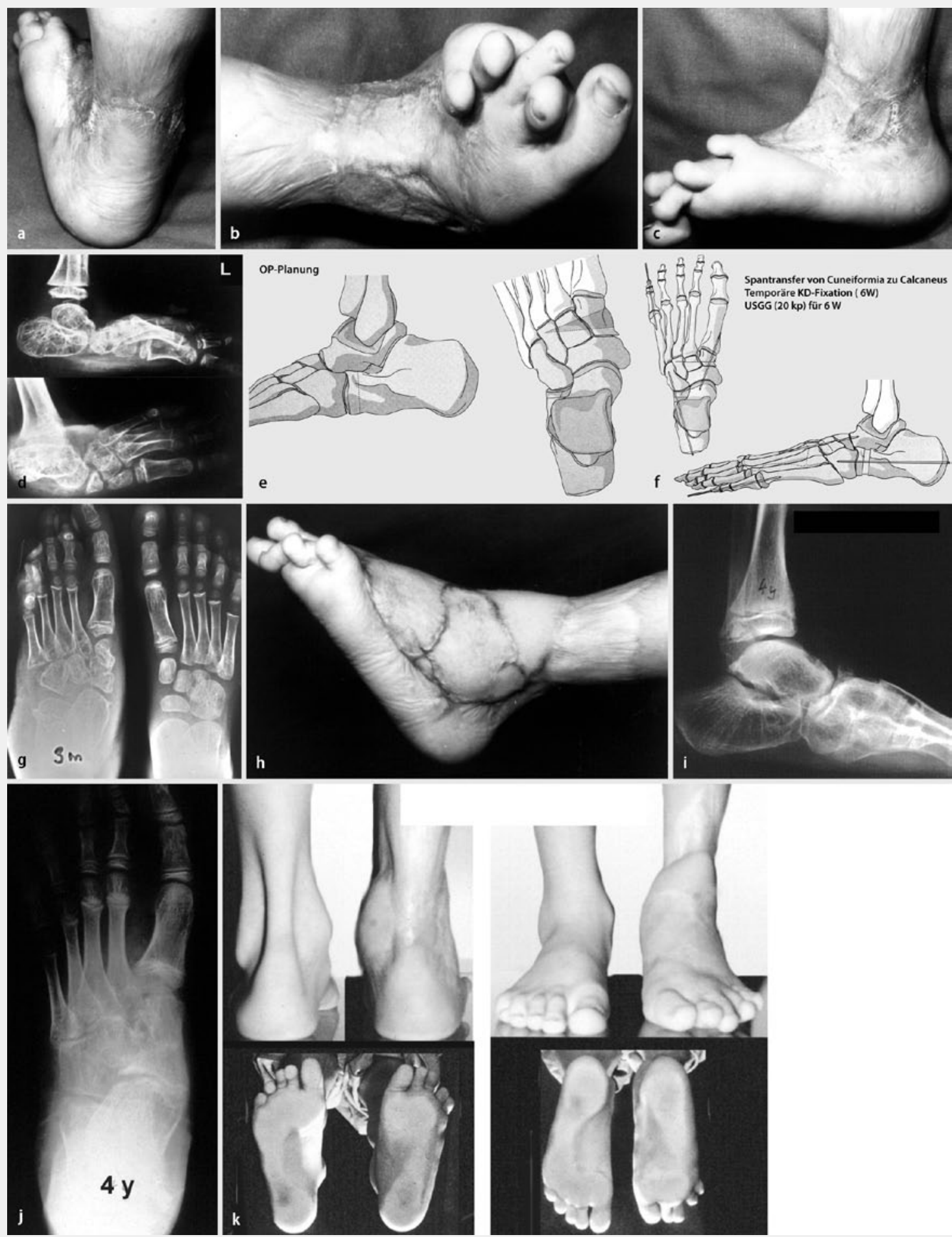

Abb. 7 A Fallbeispiel 7: Möglichkeit einer reorientierenden Ossa-cuneiformia-Osteotomie bei einem 8-jährigen türkischen Jungen, der im Rahmen eines Erdbebens verschüttet worden war und sich eine schwerste Chopart-/Lisfranc-Luxationsfraktur mit Verbrennung zugezogen hatte, a-d Klinische Bilder und präoperative Belastungsaufnahmen: hyperpronierter und abgeplatteter Fuß mit extremer Zehenfehlstellung sowie kontrakte Weichteile bei schweren Verbrennungsnarben,e,f präoperative Planungsskizzen mit geplanter zuklappender Osteotomie der 3 Ossa cuneiformia sowie zusätzlicher Verlängerung der lateralen Fußsäule mittels Evans-Osteotomie und Spaninterposition mit Achsenausrichtung der 5. Zehe. Die 3-Monats-Kontrolle $(\mathbf{g}, \mathbf{h})$ zeigte den klinisch gut eingeheilten M.-latissimus-dorsi-Lappen und die deutlich bessere Ausrichtung des Fußskelettes in der dorsoplantaren Aufsicht unter Körperlast. Das 4-Jahres-Ergebnis des zu diesem Zeitpunkt 12-jährigen Patienten ergab in den Röntgenbelastungsaufnahmen (i,j) eine nahezu normale Fußachsenausrichtung bei erhaltenem und deutlich gebessertem Chopart-Gelenk. Die klinische Kontrolle auf dem Spiegeltisch zeigte zwischenzeitlich nahezu normale Weichteilverhältnisse mit einem plantigraden Fuß und nahezu anatomisch ausgerichteten Zehen, ohne dass eine weitere Zehenkorrektur erfolgt wäre (k) 


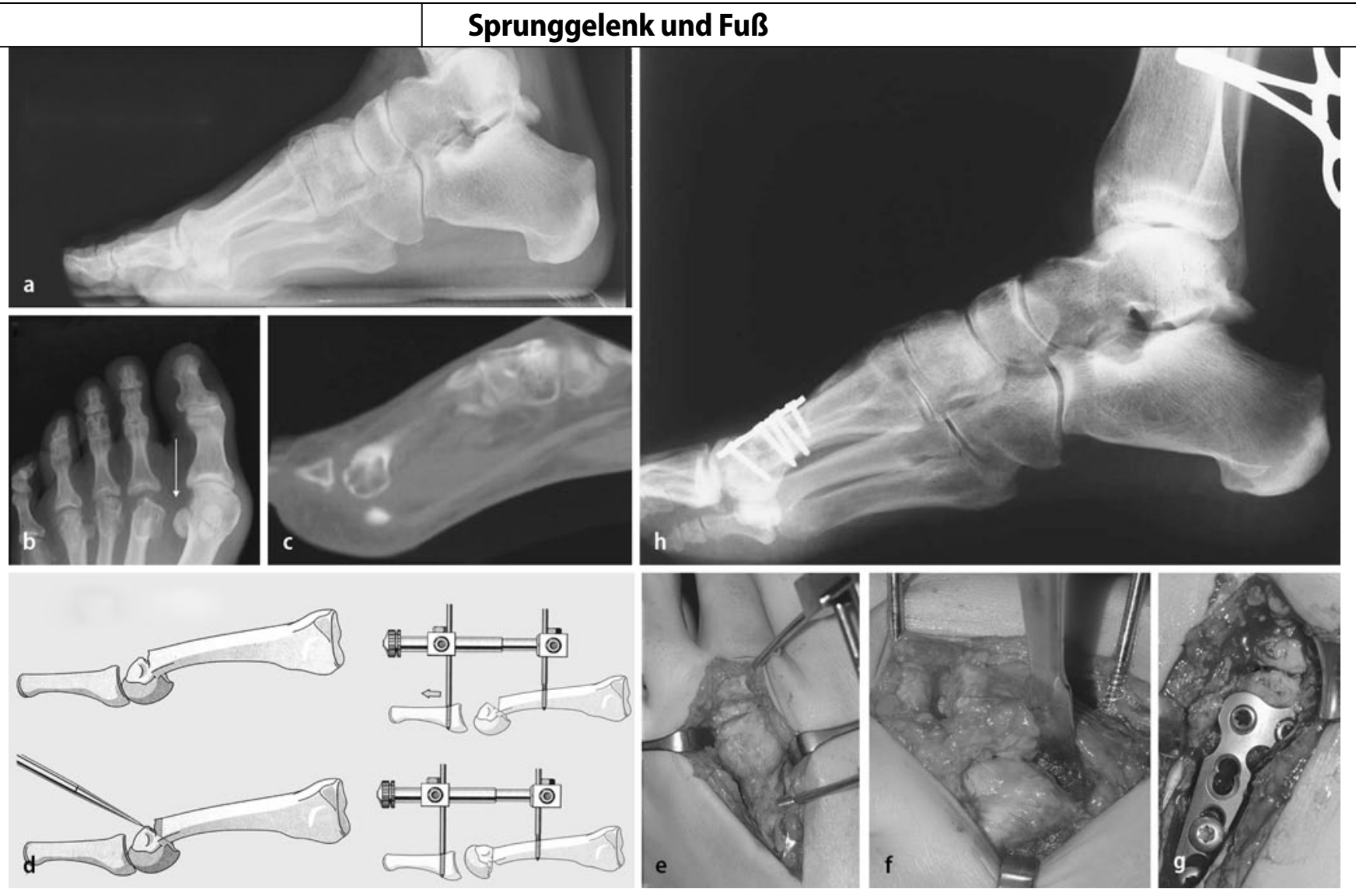

Abb. $8 \Delta$ Beispiel einer sekundären Metatarsaliaosteotomie bei erheblicher Fehlverheilung und extrem schmerzhafter Metatarsalgie durch die Fehlstellung v. a. des fehlverheilten 2. Metatarsaleköpfchens nach plantar (a,b), die insbesondere im CT (c) deutlich zu sehen war. Entsprechend der präoperativen Planung (d) wurde unter Zuhilfenahme eines Minidistraktors am Ort der Fehlstellung osteotomiert und reponiert, was auch intraoperativ $(\mathbf{e}, \mathbf{f})$ analog vorgenommen wurde. Das reponierte Köpfchen wird nach subkapitaler Osteotomie und Reposition mit einem kleinen winkelstabilen L-Plättchen korrekt in situ gehalten $(\mathbf{g}, \mathbf{h})$

schüttungstrauma im Rahmen eines Erdbebens mit Verbrennung der Haut ersichtlich. Die Operationsplanung (- Abb. 7e,f) bei dessen extrem fehlgestellten Chopart- und Lisfranc-Gelenk zeigt das geplante Realignment in unmittelbarem Anschluss nach Débridement der kontrakten Haut und vor M.-latissimus-dorsi-Transfer.

\section{Distale Metatarsaliafrakturen}

Fehlverheilte distale Metatarsaliafrakturen (- Abb. 8) nach schwerem Vorfußtrauma können gelegentlich ebenfalls eine Osteotomie erfordern. Wie in den übrigen Fällen ist auch hier eine gute präoperative Planung unabdingbar. Der Minidistraktor ist ein bedeutendes intraoperatives Repositionswerkzeug. Die heute verfügbaren, kleinen winkelstabilen Plättchen sind für die korrigierende Osteosynthese ein verlässlicher, operationstechnischer Gewinn.

\section{Fazit für die Praxis}

Sämtliche Fallbeispiele mit Korrekturosteotomien im Bereich der 5 verschiedenen Ebenen des Fußskeletts zeigen, dass durch die Reorientierung der Achsen und die direkte oder indirekte Wiederherstellung der Gelenkkongruenz in nahezu allen Fällen die posttraumatische Arthrose verhindert oder zumindest verzögert werden kann. Eine exakte Röntgendiagnostik, insbesondere durch Belastungsaufnahmen, CT und 3D-Visualisierung, intraoperative Hilfen wie Femuroder Minidistraktor sowie winkelstabile Implantate sind als hilfreiche Details zum dauerhaften Erfolg komplexer Fußchirurgie zu betrachten. Künftige Entwicklungen einer navigierten Reorientierung könnten zusätzlich sehr hilfreich sein.

\section{Korrespondenzadresse \\ Prof. Dr. H. Zwipp \\ Klinik und Poliklinik für Unfall- und Wiederherstellungschirurgie, Universitätsklinikum Carl Gustav Carus, Technische Universität Dresden, Fetscherstraße 74, 01307 Dresden Hans.Zwipp@uniklinikum-dresden.de}

Interessenkonflikt. Der korrespondierende Autor gibt an, dass kein Interessenkonflikt besteht.

\section{Literatur}

1. Romash MM (1993) Reconstructive osteotomy of the calcaneus with subtalar arthrodesis for malunited calcaneal fractures. Clin Orthop 290:157-167

2. Zwipp H (2008) Osteotomy and subtalar arthrodesis for a calcaneus malunion with nonunited sustentacular fragment. In: Marti RK, Heerwarden RJ van (eds) Osteotomies for posttraumatic deformities. Thieme, Stuttgart New York, pp 661-667

3. Zwipp H, Rammelt S (2003) Posttraumatische Korrekturoperationen am Fuß. Zentralbl Chir 128:218226

4. Zwipp H, Gavlik JM, Rammelt S (2009) Anatomische Rekonstruktion nach fehlverheilten zentralen Talusfrakturen. Fuß Sprunggelenk 7:88-96 